CLAWAR 2020: 23rd International Conference on

Climbing and Walking Robots and the Support

Technologies for Mobile Machines,

Moscow, Russian Federation, 24-26 August 2020.

https://doi.org/10.13180/clawar.2020.24-26.08.15

\title{
A FOUR-LEGGED CLIMBING ROBOT ON A FRAGILE CYLINDER
}

\author{
YURY GOLUBEV and ELENA MELKUMOVA \\ Lomonosov Moscow State University, Faculty of Mechanics and Mathematics, \\ Leninskie Gory, Moscow, 119991, Russian Federation \\ E-mail: elena_v_m@mail.ru,golubev@keldysh.ru \\ https://istina.msu.ru/profile/EVMelkumova/
}

\begin{abstract}
The problem of the equilibrium of a multi-legged walking robot on the surface of a brittle straight circular rough cylinder is investigated. Each of the legs has one point in contact with the cylinder with Amonton-Coulomb friction and for two support points, with rolling friction. Numerically and analytically obtained possible regions of contact points on the cylinder for which there is a solution to the kinetostatics problem on the cylinder. This task has analogies to the problems of holding a cylindrical object by the fingers of a robot arm or a robot resting on an arbitrary surface, the legs of which are suspended on the body on the surface of the cylinder. The robot can climb on the cylinder with two legs located on the same diameter at the base of the cylinder. And due to dry friction with four legs located on opposite sides from the robot center of mass or point $\mathrm{C}$ introduced into the dynamics. The oscillations of the cylinder on the cylinder in the vicinity of a stable equilibrium position serve as an analogue of the problem. The cylinder lies on one finger of the hand of a humanoid robot perpendicular to it, adheres to the ends of the other three fingers. Similarly holds a glass.
\end{abstract}

\section{Introduction}

In this paper, we discuss the problem of walking robot dynamics on one-side constraint. While the general walking robot motion on a plane was analyzed in detail in the work ${ }^{1}$ the case of the dynamics on a curved surface is far more complicated. Model dynamics and control problems were considered in the work. ${ }^{2}$ Equilibrium conditions for a solid on a rough plane was considered in the work. ${ }^{3}$ Walking robot parameters optimization for the motion in tubes was considered in the work. ${ }^{4}$ The special case of a robot with eight legs whose up porting points are restricted to the inner surface of a tube was considered in the work. ${ }^{5}$ In the work ${ }^{6}$ control of the movement of the manipulator along with the connection was disscused. In the present work, we consider the more general case of a robot with four arbitrary supporting points on a rough cylinder and on a curved surface. And discussed the problem of transfer by a manipulator with a four-finger grasp of a rough cylinder.

\section{The problem of climbing the cylinder}

We study the problem of a walking robot consisting of a body, $l$ hands with $m$ fingers and $n$ feet resting on a straight circular rough cylinder, of radius $\rho$, at $n+m$ support points. It is assumed that the hands, fingers and feet of the robot make a given movement relative to the body of the robot. Each of the fingers have one point in contact with the cylinder with Amonton Coulomb friction and for two support points, with rolling friction. Numerically and analytically obtained are possible regions of contact points on the cylinder for which there is a solution to the kinetostatics problem when transferring the cylinder with four fingers. The robot can hold the cylinder with two fingers located on the same diameter at the base of the cylinder. And due to dry friction with four fingers located on opposite sides from the center of mass of the cylinder or point $C$ introduced into the dynamics.

We number the reference points. If the reference point is one, then the reference set is a 
point. In the case of two points, the traversal of the graph is a segment. For three points, a reference triangle. Four points can be traversed along the supporting convex quadrangle. Or save on a detour with three segments of the letter $\mathrm{x}$ with a cover. These graphs reflect the relationship of internal forces between pairs of points. And if you add to them the force applied in the center of mass, then you can follow them using the graph in the convex pentagon or drawn on connecting all the pairs of points of the star in a continuous connection. Note that the support polygons are spatial. And spatial x-shaped stars or stars constructed with roundabouts with intersection can be more convenient than supporting polygons, even generalized to spatial ones. Five, six and eight fulcrum points give either a convex pentagon, and then, respectively, or stars. This justifies five fingers on a person's hand, six legs of a bug and eight spiders.

An object grasping problem is equivalent to the problem of the walking robot with $n$ legs. ${ }^{7,8}$ The figure 1 shows the case of four points in support and to the right one of
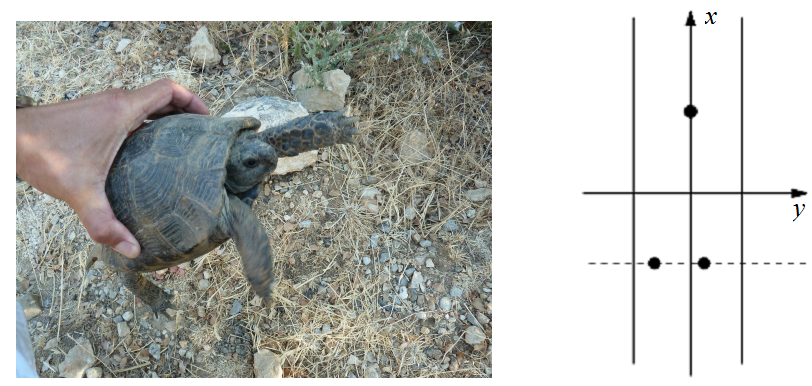

Fig. 1. Division of a reference set into two subsets.

the possible support triples. The thumb is at one end of the diameter of the cylindrical shell of the turtle. The remaining fingers are in the neighborhood of the solution when two support points are located on one straight line orthogonal to the axis of the cylinder. ${ }^{9}$ In co-authorship with V.V. Koryanov, we considered the turning of a six-legged walking robot into working condition from an emergency situation. The turtle, for example, flips over by pushing it off the surface. An alternative method could be the help of another walking robot with a four-finger manipulator. Unless of course the robot is not able to perform the tip-top spinning trick.

Let the point $O$ is an origin fixed in absolute space. Suppose that robot arms fingers accomplish the desired motion with respect to the body of the robot. Using general dynamics theorems to describe the cylinder motion, we obtain six different equations for the cylinder dynamics from the momentum and angular momentum theorems. Among them, there are three equations of the body translation with point $A$ and another three describe body rotation about point $A$. For prescribed motion be realized then reaction in $m$ footholds should satisfy following kinetostatic equations: ${ }^{9}$

$$
\sum_{i=1}^{m} \tilde{\mathbf{R}}_{i}=-\tilde{\boldsymbol{\Phi}}, \quad \sum_{i=1}^{m} \tilde{\mathbf{r}}_{i} \times \tilde{\mathbf{R}}_{i}=-\tilde{\mathbf{M}}
$$

where $\tilde{\mathbf{R}}_{i}$ is reaction component, $\tilde{\mathbf{r}}_{i}$ corresponds to the $i$-th finger supporting point vector, $\tilde{\boldsymbol{\Phi}}$ is the sum of the external active forces plus time derivative of the desired momentum, and $\tilde{\mathbf{M}}$ is the sum of external active forces momentum and time derivative of desired angular momentum with respect to the point $O$. In two vector equations in (1), the former corresponds to the momentum of the object (and is equivalent to three scalar equations when projected onto the basis vectors), while the latter defines the desired change of the angular momentum. 
Assuming that $\tilde{\boldsymbol{\Phi}}$ is orthogonal to $\tilde{\mathbf{M}}$, we obtain that the system $\{\tilde{\boldsymbol{\Phi}}, \tilde{\mathbf{M}}\}$ can be also used at the point $C$

$$
\tilde{\mathbf{r}}_{C} \times \tilde{\mathbf{\Phi}}=\tilde{\mathbf{M}}, \quad \tilde{\mathbf{r}}_{C}=-\frac{\tilde{\mathbf{M}} \times \tilde{\mathbf{\Phi}}}{\tilde{\Phi}^{2}}, \quad \tilde{\Phi}=|\tilde{\mathbf{\Phi}}|,
$$

where $\tilde{\mathbf{r}}_{C}$ is the vector $\mathbf{O C}$, and $C$ corresponds to the point at which the resultant of the reactions is acting. The fragility of the cylinder means a restriction on the modulus of the normal components of the reactions: $\tilde{N}_{i} \leq a$.

A further problem of reactions distribution $\tilde{\mathbf{R}}_{i}$ in some fixed point of time is investigated by the proposal that force $\tilde{\boldsymbol{\Phi}}$ is acting at the point $\tilde{\mathbf{r}}_{C}$ and force moment there is zero. Motion equations (1) for finding reactions of fingers prescribed motion can be transformed: ${ }^{10}$

$$
\sum_{i=1}^{m} \tilde{\mathbf{R}}_{i}=\tilde{\boldsymbol{\Phi}}, \quad \sum_{i=1}^{m} \tilde{\mathbf{r}}_{i} \times \tilde{\mathbf{R}}_{i}=\tilde{\mathbf{r}}_{C} \times \tilde{\boldsymbol{\Phi}} .
$$

For example point $C$ can be the grasping object center of mass.

Assuming that the robot footholds are on the surface of a rough cylinder of radius $\rho$ with a friction coefficient $k$, we introduce the coordinate system $O x y z$ such that the axis $O x$ is directed along the cylinder axis (so that the projection of $\tilde{\boldsymbol{\Phi}}$ on the axis $O x$ is negative see Fig. 2.), the axis $\mathrm{Oz}$ is parallel to the vector $\tilde{\boldsymbol{\Phi}}$, and the angle between the cylinder axis and the vector $\tilde{\boldsymbol{\Phi}}$ is $\alpha .{ }^{11}$
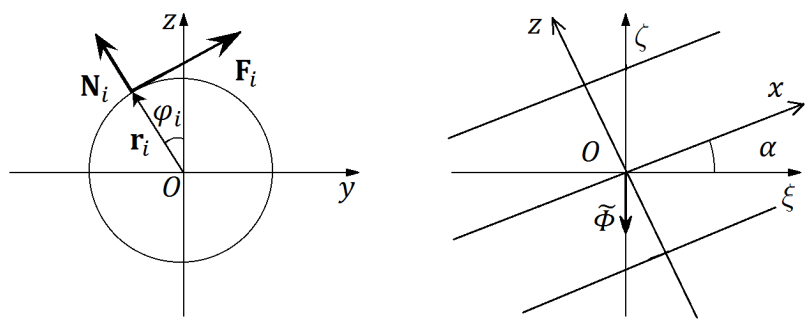

Fig. 2. Fragile cylinder.

The problem of finding the reaction forces (2) is similar to the foothold reactions distribution problem for walking robot, when the footholds are on the external surface of a rough inclined cylinder where the axis has an angle $\alpha$ with respect to the vector $\tilde{\boldsymbol{\Phi}}$.

In the coordinates $O x y z$ we define $\tilde{\mathbf{R}}_{i}=\left(\tilde{R}_{i}^{x}, \tilde{R}_{i}^{y}, \tilde{R}_{i}^{z}\right), \tilde{\mathbf{r}}_{C}=\left(\tilde{x}_{C}, \tilde{y}_{C}, \tilde{z}_{C}\right)$, and $\tilde{\boldsymbol{\Phi}}=(-\tilde{\Phi} \sin \alpha, 0,-\tilde{\Phi} \cos \alpha), i=1, \cdots, m$. In case of a one-sided surface, and the grasp inside the cylinder, we have additional restrictions on normal reactions $\tilde{N}_{i}:{ }^{12}$

$$
\tilde{N}_{i}=\tilde{\mathbf{R}}_{i} \cdot \mathbf{e}_{\nu}^{i} \geq 0,
$$

where $\mathbf{e}_{\nu}^{i}$ is an external normal to $i$-th supporting point on the cylinder, while the tangential components are given by $\tilde{\mathbf{F}}_{i}=\tilde{\mathbf{R}}_{i}-\tilde{N}_{i} \mathbf{e}_{\nu}^{i}$.

For the reactions to be in the friction cones (2), we have following inequalities:

$$
\left|\tilde{\mathbf{F}}_{i}\right| \leq k \tilde{N}_{i},
$$

i.e. the tangential reactions $\tilde{\mathbf{F}}_{i}$ are restricted by Coulomb limiting friction value. When $\tilde{\mathbf{F}}_{i}$ exceeds this limiting value, the robot legs and arms begin to slide along a surface.

The reaction distribution problem then reduces to the solution of equations (2), and inequalities (3), (4), for reactions limited to the friction cones. The restricted motion can only be realized if the solution of system (2)-(4) does exist.

The same inequalities are for walking robot on the cylinder. ${ }^{13}$ If the grasp is out the cylinder this inequalities (3) have opposite signs. 
For example if $m$ is even, and one of each par of the supporting points is on and another is in the thin surface such that we consider them like one geometrical point. Then we need only inequalities (4).

For $\mathbf{r}_{i}=\tilde{\mathbf{r}}_{i} / \rho=\left(x_{i}, y_{i}, z_{i}\right)$, in the cylinder coordinate: $\mathbf{r}_{i}=\left(x_{i},-\sin \varphi_{i}, \cos \varphi_{i}\right), \mathbf{e}_{i}^{\nu}=$ $=\left(0,-\sin \varphi_{i}, \cos \varphi_{i}\right), N_{i}=\tilde{\mathbf{N}}_{i} / \tilde{\Phi}=\left(0,-N_{i} \sin \varphi_{i}, N_{i} \cos \varphi_{i}\right)$, where $\varphi_{i}$ is the angles between axis $O z$ and cylinder normal $\mathbf{e}_{i}^{\nu}$. We define $\mathbf{e}_{x}$ as the unitary vector in the $O x$ axis, while $\mathbf{e}_{i}^{\tau}=\left(0, \cos \varphi_{i}, \sin \varphi_{i}\right)$ as the tangential to the cylinder. Then the tangential reaction: $\mathbf{F}_{i}=$ $=\left(F_{i}^{x}, F_{i}^{y z} \cos \varphi_{i}, F_{i}^{y z} \sin \varphi_{i}\right)$, where $F_{i}^{x}=\mathbf{F}_{i} \cdot \mathbf{e}_{x}, F_{i}^{y z}=\mathbf{F}_{i} \cdot \mathbf{e}_{i}^{\tau}, \quad \mathbf{R}_{i}=\tilde{\mathbf{R}}_{i} / \tilde{\Phi}=\left(R_{i}^{x}, R_{i}^{y}, R_{i}^{z}\right)$, $\mathbf{r}_{C}=\tilde{\mathbf{r}}_{C} / \rho=\left(x_{C}, y_{C}, z_{C}\right)$.

Projecting onto Oxyz the first and the second vector equations (2) we obtain

$$
\begin{gathered}
\sum_{i=1}^{m} F_{i}^{x}=\sin \alpha \\
\sum_{i=1}^{m}\left(N_{i} \sin \varphi_{i}-F_{i}^{y z} \cos \varphi_{i}\right)=0 \\
\sum_{i=1}^{m}\left(N_{i} \cos \varphi_{i}+F_{i}^{y z} \sin \varphi_{i}\right)=\cos \alpha \\
\sum_{i=1}^{m} F_{i}^{y z}=-y_{C} \cos \alpha \\
\sum_{i=1}^{m}\left(x_{i} N_{i} \cos \varphi_{i}+x_{i} F_{i}^{y z} \sin \varphi_{i}-F_{i}^{x} \cos \varphi_{i}\right)=x_{C} \cos \alpha-z_{C} \sin \alpha \\
\sum_{i=1}^{m}\left(x_{i} N_{i} \sin \varphi_{i}-x_{i} F_{i}^{y z} \cos \varphi_{i}-F_{i}^{x} \sin \varphi_{i}\right)=y_{C} \sin \alpha .
\end{gathered}
$$

Let $p=R_{1}^{x}-R_{2}^{x}$. We further define the coordinate differences, and the supporting points difference of angles of axis $O z$ are $\Delta x=x_{2}-x_{1}, \Delta y=y_{2}-y_{1}, \Delta z=z_{2}-z_{1}, \Delta \varphi=$ $=\varphi_{2}-\varphi_{1}$. We then project system (2) onto the axes Oxyz. For arbitrary surface we find that the second equation of (2) (corresponding to the moment) has the skew-symmetric matrix with respect to the component $R_{i}^{x}$. These are 2 independent equation, while the third equation corresponds to the restriction of the point $C$ to the plane containing the two footholds. As a result, the system (2) yields 5 independent equations and a restriction.

\section{A four-finger grasp}

During the robot motion one, two, three and four-supporting points phases are changed. For example, Australian lizards - yellow-bellied three-toed skinks (saiphos equalis). Or a horse with four legs.

First, we consider the one-supporting phase of the grasp. Let $m=1$, then the motion existing condition is reaction is equal to force $\boldsymbol{\Phi}$ and supporting point and the point $C$ are on the line along $\boldsymbol{\Phi}$, while the angle between $\boldsymbol{\Phi}$ and the normal do not exceed the friction angle.

If the grasp inside the surface then point $C$ is under the surface. In the opposite case the grasp is under the surface. Then point $C$ is inside the surface. Or if one finger out the cylinder, the center mass of an object is up the finger. And the angle between the weight and the normal not exceed friction angle.

If $m$ is even. And one of each par of the supporting points is on and another is in the thin surface such that we consider them like one geometrical point. Then it does not matter where the point $C$ is on the line. 

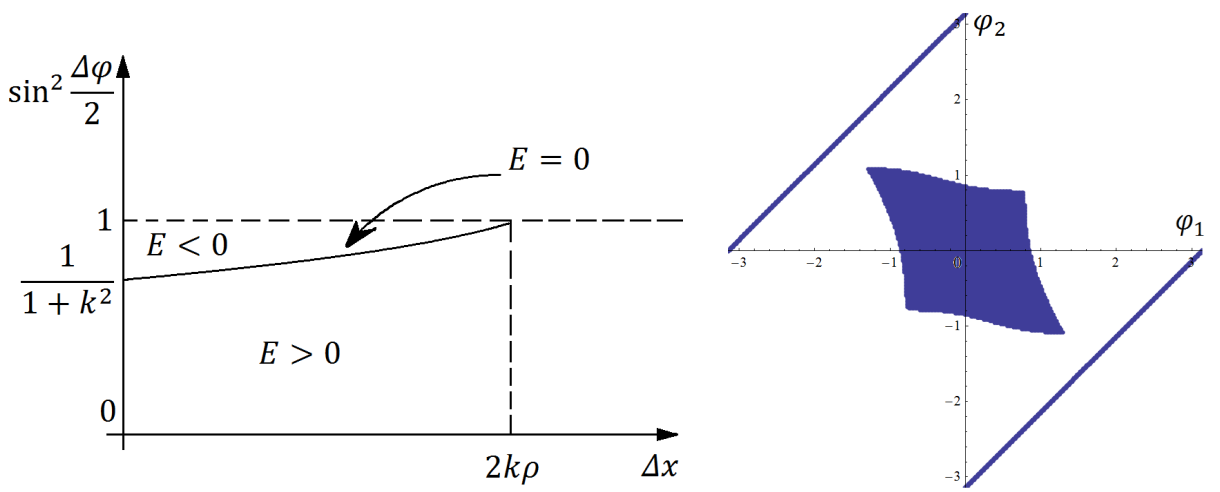

Fig. 3. The analytical and the numerical parameter diagrams.

Let $n=2$, and $x_{1} \neq x_{2}$. Then $p=F_{2}^{x}-F_{1}^{x}$, and from (2):

$$
\begin{gathered}
F_{1}^{x}=(\sin \alpha+p) / 2, \quad F_{2}^{x}=(\sin \alpha-p) / 2, \\
N_{1}=\frac{-p \sin ^{2} \frac{\Delta \varphi}{2}+\left(x_{2}-x_{C}\right) \cos \varphi_{1} \cos \alpha}{\Delta x}+N_{1}^{\alpha}, \\
N_{2}=\frac{-p \sin ^{2} \frac{\Delta \varphi}{2}+\left(x_{C}-x_{1}\right) \cos \varphi_{2} \cos \alpha}{\Delta x}+N_{2}^{\alpha}, \\
F_{1}^{y z}=\frac{-p \sin \Delta \varphi+2\left(x_{2}-x_{C}\right) \sin \varphi_{1} \cos \alpha}{2 \Delta x}+F_{1}^{(y z) \alpha}, \\
F_{2}^{y z}=\frac{p \sin \Delta \varphi+2\left(x_{C}-x_{1}\right) \sin \varphi_{2} \cos \alpha}{2 \Delta x}+F_{2}^{(y z) \alpha}, \\
\tan \alpha=\frac{\Delta x\left(\sin \varphi_{2}+y_{c}\right)+\left(x_{C}-x_{2}\right) s_{21}}{y_{C} c_{21}+z_{C} s_{21}-\sin \Delta \varphi},
\end{gathered}
$$

where $N_{i}^{\alpha}$ and $F_{i}^{y z}$ are the functions of $x_{i}, \varphi_{i}, y_{C}$ and $z_{C}$.

The conditions (4) can be displayed in the form

$$
E p^{2}+B_{1} p+C_{1} \leq 0, \quad E p^{2}+B_{2} p+C_{2} \leq 0,
$$

where

$$
E=(\Delta x)^{2}+\sin ^{2} \Delta \varphi-4 k^{2} \sin ^{4}\left(\frac{\Delta \varphi}{2}\right),
$$

$B_{i}, C_{i}$ are the functions of $x_{i}, \varphi_{i}, x_{C}, y_{C}$ and $z_{C}$.

The boundaries between different regimes can be determined analytically. For example, in the case of $E<0$, the solution exists, and can be obtained analytically, as shown in Fig. 3, on the left. Note that in this case it's limited to the range $\Delta x \leq 2 k \rho$. In contrast to this behavior, for $E \geq 0$ there is no such restriction and an additional step is required to address the question of the existence of the solution. At the point $(0,0)$ we find $E=0$, which means that two footholds are orthogonal to the cylinder axis. Here, two possible solutions are either identical, or limited to a single diameter. In the latter case, point $C$ and the reaction have to be in one plane, parallel to force $\Phi$, and the problem has a solution. For the desired legs or fingers configurations and given point $C$, the problem can be solved numerically. In Fig. 3, on the right, we present the numerical solution for the example when $x_{2}=-x_{1}=\rho=$ $=k=1$. Note that in this case $E>0$.

When $E=0$, the reaction distribution problem reduces to the linear inequalities for the parameter $p$. Specifically, the condition (7) was analyzed in two cases, when $E=0$ and 

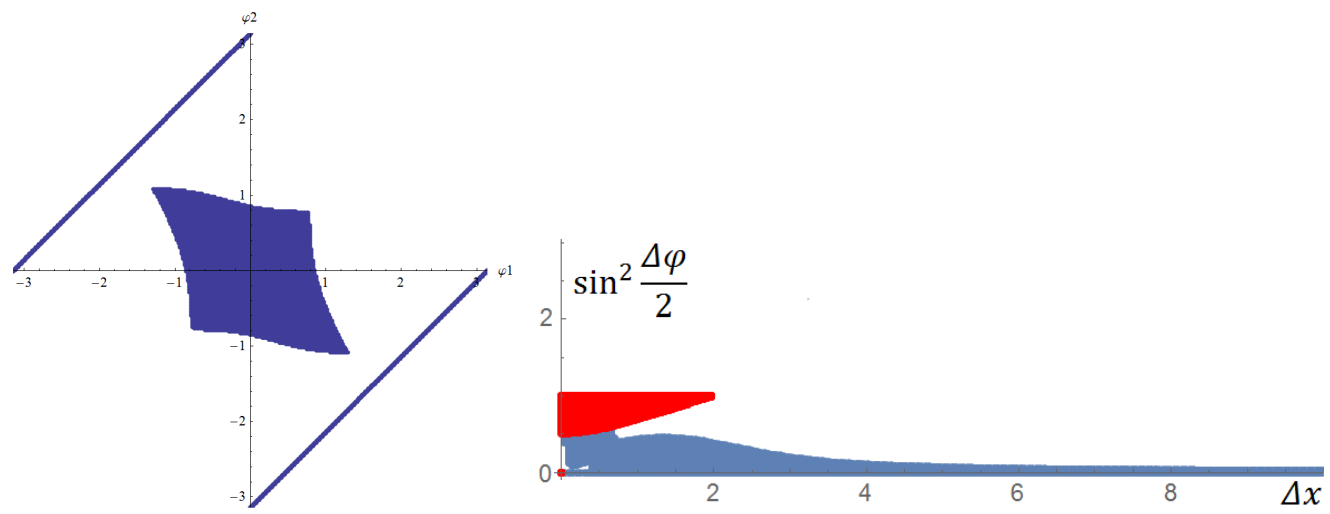

Fig. 4. The case of $\alpha=0, x_{2}=-x_{1}=\rho=k=1$.

$E>0$, and when the solution of the problem does exist, the solutions were shown in the plot Fig. 4.

For $E>0$, we need to consider two conditions. First is the restriction on the determinants $D \geq 0$, while the second is the requirement of a non-empty intersection of the set of point of the intervals between the roots of quadratic equations. From this plot, we see that, if two points are on one diameter, then the solution of the reaction distribution problem exist. The two lines in the plot, correspond to $\varphi_{1}=\varphi_{2}+\pi$ or $\varphi_{1}=\varphi_{2}-\pi$. The rhombus form represents the requirement on the determinants $D_{i} \geq 0$, while additional conditions further restrict the range.

In Fig. 4 on the right we present the results for $E>0$ and $E \leq 0$, when $x_{2}=-x_{1}$, $\varphi_{2}=-\varphi_{1}$ and shows the case of $\alpha=\pi / 4$. The figures for $\alpha=0$ and increased to $\pi / 2$ are shown in the work. ${ }^{14}$ Note that when $\alpha=\pi / 2$, the solutions exist only for diametrical footholds.
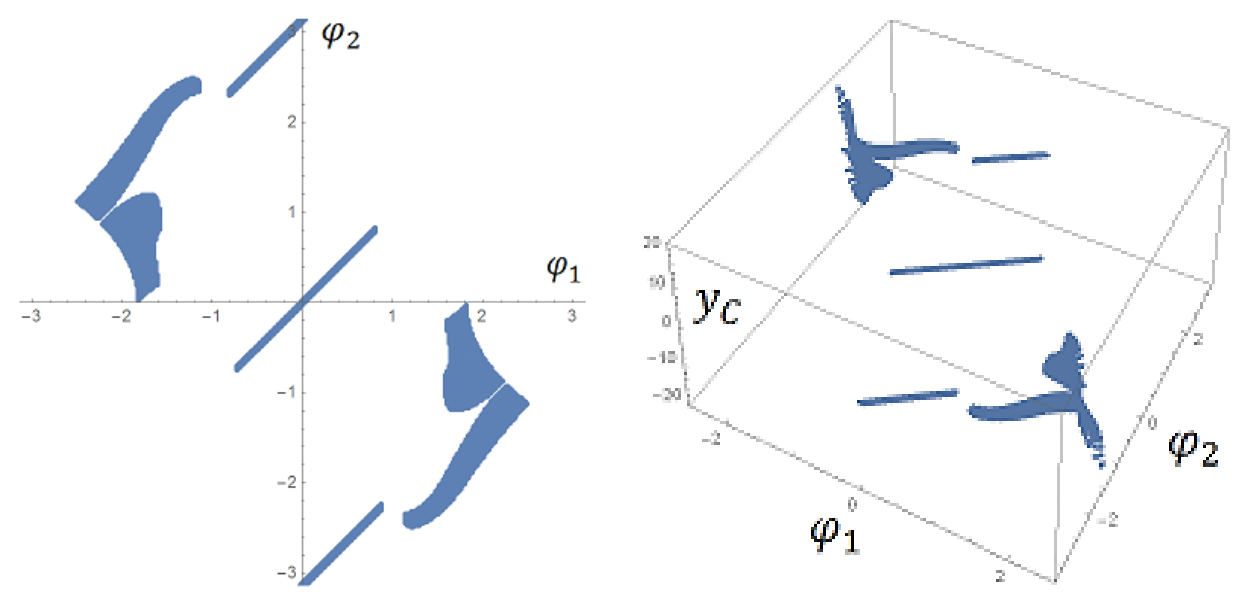

Fig. 5. The parameter range for $\alpha=\pi / 3 ; x=1,1$.

For two-finger robot when $E$ is negative, the solution exist, and obtained analytically. ${ }^{16}$ Using numerical simulations we explain the reaction distribution problem existing and build this problem solution existing fields for given footholds and point $C$ position. For example, for two-foothold phase, we consider symmetric, about point $C$, along and orthogonal cylinder axis, robot configurations. For first of these configurations examined three cases with 
nonnegative $E$ coefficient, for distance x, between point $\mathrm{C}$ and footholds: 0,9; 1 and 1,1 at $\rho$ and $k$ equal $1, \alpha$ from 0 to $\pi$ (in all 13 different values cylinder inclination angles). Reactions distribution problem solution existing fields constructed on the two angles plane, correspond to footholds projections on the cylinder base and three dimensional fields which supplement this plane by point $\mathrm{C} z$-coordinate altitude. When $x$ equals to 1,1 for $\alpha$ equals $\pi / 3$ in three-dimensional fields observed bundles of separate points, Fig. 1 . That means that the point $C$ altitude position more harsh change while changing the angles.

When $\alpha$ equals to $0, x$ equals to 1 , the field consist of three separate situated subregions. On the angle plane each of pair parallel lines corresponds to support on the cylinder diameter plane section contained point $C .{ }^{14}$ There is connected field between these lines. It contains the line segment corresponding to the angles equality, robot supported above on the line which is parallel to cylinder axis and satisfy force direction deviation restriction. The indicated segment on the plot disappear when $\mathrm{x}$ equals to 0,9 for $\alpha$ equals $\pi / 4$, and at increasing $x$, later, for $4 \pi / 9$. It corresponds to the robot beginning sliding down the cylinder. ${ }^{15}$ When $x$ equals to 1,1 for $\alpha$ equals $\pi / 3$ in three-dimensional fields observed bundles of separate points. That means that the point $C$ altitude position more harsh change while changing the angles.

There is an analogy of supporting on a cylinder and on one or two planes. Let speak about static stability of walking apparatus with support on two unilateral planes. For example a smooth planes in homogeneous gravity field. The static-stability conditions for a walking apparatus with supporting points at the interior surface of a smooth horizontal cylinder and on two smooth planes was studied in ${ }^{8}$ on the right.
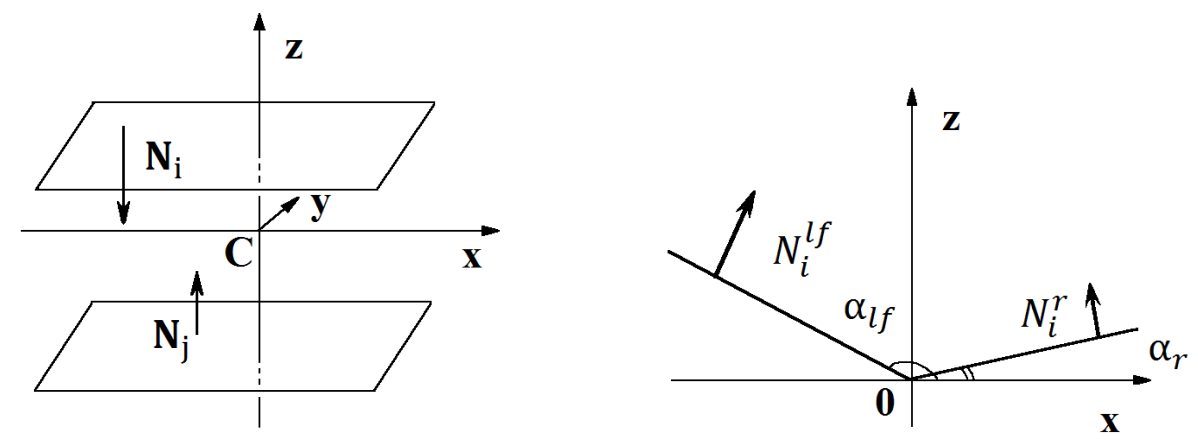

Fig. 6. Two planes.

\section{Conclusion}

If the cylinder is fragile, then three legs may not be enough. The robot can walk on fragile cylinder with four legs located on the outer surface of the upper half cylinder. In this case, the load on four footholds is distributed more evenly, so that the values of normal reactions do not exceed the specified value $a$. For example, let one of the points of support and the center of mass of the robot be located in the vertical plane containing the axis of the cylinder, and the other two points of support in the plane orthogonal to the axis of the cylinder, while the robot center of mass is located inside the support triangle. Similarly, if the robot is on its inner surface, then four support points should be located on the inner half of the cylinder. Four footholds may be located in a vertical plane containing the axis of the cylinder. They can also be located symmetrically in the vicinity of the section of the cylinder passing through the point $C$. The four-legged robot can go gallop and whether to rearrange its legs in pairs. 


\section{Acknowledgments}

This work was supported by the Russian Foundation for Basic Researches (grant no. $19-$ $01-00123 \mathrm{~A})$.

\section{References}

1. D. E. Okhotsimski and Yu. F. Golubev, Mechanics and Motion Control of Automatic Locomotion Apparatus. Moscow, Russia: Nauka, 1984. (In Russian.)

2. V. V. Beletski. Two-legged Locomotion. Model Dynamics and Control Problems. Moscow, Russia: Nauka Publishers, 1984. (In Russian.)

3. F. L. Chernousko. Equilibrium conditions for a solid on a rough plane. Izvestiya RAN, ser. Solid Mechanics, No. 6, pp. 6-17, 1988

4. F. L. Chernousko and N. N. Bolotnik. Walking robot parameters optimization for the motion in tubes. Izvestiya RAN, ser. Solid Mechanics, No. 6, pp. 27-41, 1995.

5. F. Pfeiffer et al., Optimization of Structural Parameters and Gates of a Pipe Crawling Robot, IUTAM Symposium on Optimization of Mechanical Systems, D. Bestli; W. Schiehlen. IAF, Kluwer Academic Publisgers, 1996, pp. 231-238.

6. Lensky A.V., Lizunov A.V., Mozhzhevelov S.B. and etc. Control of the movement of the manipulator along the connection // Bulletin of the USSR Academy of Sciences. Solid mechanics. - 1987. - No. 5. - S. 4149.

7. Yu. F. Golubev, The Fundamentals of Theoretical Mechanics, Russia: Moscow State University, 2019. (In Russian.)

8. Yu. F. Golubev and E. V. Melkumova, Static-Stability Conditions for a Walking Apparatus in Horizontal Cylinder and on Two Planes. Journal of Computer and Systems Sciences International. Robotics. Pleiades Publishing, Ltd., vol. 38, 2, pp. 278-284, 1999. (Translated from Izvestiya Akademii Nauk. Teoriya i Sistemy Upravleniya, 2, pp. 116-122, 1999.)

9. Yu. F. Golubev and E. V. Melkumova, On Stability of Equilibrium Positions for Walking Robot in a Smooth Horizontal Tube. Proc. of the Third International Conference on Climbing and Walking Robots, Madrid, Spain, pp. 433-440, 2000.

10. Yu. F. Golubev and E. V. Melkumova, Walking Robot Dynamics on a Rough Inclined Cylinder ESMC-2012 - 8th European Solid Mechanics Conference, Graz, Austria, July 9-13, 2012, Book of Abstracts, Editors: Gerhard A. Holzapfel and Ray W. Ogden. On CD 63677.pdf. pp. 1-2.

11. Yu. F. Golubev and E. V. Melkumova, Prescribed Motion of a Two-legged Walking Robot on a Rough Cylinder. Proc. of the 2016 International Conference Stability and Oscillations of Nonlinear Control Systems (Pyatnitskiys Conference), Moscow, Russia, pp. 1-4.

12. Yu. F. Golubev and E. V. Melkumova, The existence of solution for robot motion on a cylinder pipe. All-Russian Meeting of University Lecturers and Department Heads on Theoretical Mechanics, Robotics and Mechatroinics. Conference Materials. Edited by V. A. Samsonov. Makhachkala, Russia: Publishing center Master (Didkovskaya N.V.), 2016, pp. 30-33. (In Russian.)

13. Yu. F. Golubev and E. V. Melkumova, Footholds Admissible Areas Structure Properties for a Two-legged Walking Robot on an Inclined Cylinder. In The Lomonosov Readings. Mechanics Section. (Lomonosov Moscow State University, Moscow, Russia, 2017), pp. 64-65. (In Russian.)

14. Yu. F. Golubev and E. V. Melkumova, Footholds Admissible Areas Structure of a Two-legged Walking Robot on an Inclined Cylinder. Abstracts. International Scientific Conference. Fundamental and Applied Problems of Mechanics, Dedicated to the 170th Anniversary of a Distinguished Russian Scientist N. E. Zhukovsky. Bauman Moscow State Technical University, Moscow, Russia, 2017, pp. 83-84. (In Russian.)

15. Golubev Yu.F., Melkumova E.V. Two-legged walking robot prescribed motion on a rough cylinder // AIP Conference Proceedings. 2018. Vol. 1959, no. 030009. P. 03000910300098. DOI: $10.1063 / 1.5034589$.

16. Golubev Yu.F., Melkumova E.V. Transfer of a fragile cylinder by a three-finger manipulator // XII All-Russian Congress on Fundamental Problems of Theoretical and Applied Mechanics: a collection of works in 4 volumes. D23 T. 1: General and Applied Mechanics. Ufa: RIC of BashSU. 780 p. - T. 1. - 2019. - S. 543-545. ISBN 978-5-7477-4951-1. DOI: 10.22226 / 24103535-2019-congress-v1. 